\title{
Twin supersymmetry
}

\author{
Adam Falkowski, ${ }^{1,2, *}$ Stefan Pokorski, ${ }^{2, \dagger}$ and Martin Schmaltz ${ }^{3, \ddagger}$ \\ ${ }^{1}$ CERN Theory Division, CH-1211 Geneva 23, Switzerland \\ ${ }^{2}$ Institute of Theoretical Physics, Warsaw University, Hoża 69, 00-681 Warsaw, Poland \\ ${ }^{3}$ Physics Department, Boston University, Boston, Massachusetts 02215, USA
}

(Received 21 April 2006; published 2 August 2006)

\begin{abstract}
We construct an extension of the minimal supersymmetric standard model in which superpartners can naturally be heavier than the electroweak scale. This "little hierarchy" of scales is stable because the Higgs arises as a pseudo-Nambu-Goldstone boson in the breaking of an accidental $S U(4)$ symmetry of the Higgs sector. Supersymmetry and the global symmetry combine to forbid logarithmically divergent oneloop contributions to the Higgs mass. The accidental symmetry follows from a simple twin parity which exchanges the $S U(2)$ sectors in the $S U(3)_{C} \times S U(2)_{L} \times S U(2)_{R} \times U(1)_{X}$ gauge group.
\end{abstract}

DOI: 10.1103/PhysRevD.74.035003

PACS numbers: 12.60.Jv

\section{INTRODUCTION}

Supersymmetry is a very attractive scenario for physics at the $\mathrm{TeV}$ scale. Unfortunately, its simplest implementation, the minimal supersymmetric standard model (MSSM), requires fine-tuning of parameters once experimental constraints are imposed. The problem can be summarized as follows: In the MSSM scalar superpartners and the Higgs scalars are on equal footing, leading us to expect that the superpartner mass scale $M_{\mathrm{SuSY}}$ is equal to the Higgs mass and the electroweak scale $M_{W} \sim 100 \mathrm{GeV}$. Clearly, this expectation is not borne out in Nature as shown by direct searches, limits from precision electroweak and flavor constraints, and from the lower bound on the mass of the Higgs boson. Instead the data prefer that the superpartners are heavier than the electroweak scale. The next-most natural expectation might be $M_{\mathrm{SUSY}} \sim 4 \pi M_{W}$, and we must ask if this modest hierarchy of scales is stable under radiative corrections.

In the MSSM, the largest radiative correction to the Higgs soft mass is due to top/stop loops

$$
\delta m_{H}^{2} \simeq-\frac{3 y_{t}^{2}}{8 \pi^{2}} m_{\tilde{t}}^{2} \log \left(\frac{\Lambda_{\mathrm{UV}}^{2}}{m_{\tilde{t}}^{2}}\right)
$$

where $y_{t}$ is the top Yukawa coupling, $m_{\tilde{t}}$ is the stop mass (for simplicity, we consider degenerate left- and righthanded stops and ignore stop mixing), and $\Lambda_{\mathrm{UV}}$ is the high energy scale at which the soft masses are generated. We see that for large values of $\Lambda_{\mathrm{UV}}$, for example $\Lambda_{\mathrm{UV}} \sim$ $M_{\text {Planck }}$ in gravity mediation, the suppression due to the loop factor is canceled by the large logarithm, and a hierarchy between superpartner masses and the Higgs mass is unstable.

\footnotetext{
*Electronic address: adam.falkowski@cern.ch

${ }^{\dagger}$ Electronic address: pokorski@fuw.edu.pl

‡Electronic address: schmaltz@bu.edu
}

Improving naturalness of supersymmetric theories with heavy superpartners therefore requires removing the large logarithm. ${ }^{1}$ Two possibilities suggest themselves. One is to lower the scale $\Lambda_{\mathrm{UV}}$ down to the $1-100 \mathrm{TeV}$ range. This can be achieved in scenarios in which supersymmetry breaking is mediated at a low scale, such as gauge mediation or theories with large extra dimensions. Effectively small $\Lambda_{\mathrm{UV}}$ may also be a consequence of an interplay between gravity and anomaly mediation, as recently pointed out in [2]. But even in a theory in which such a low mediation scale is realized, one still wonders what makes the Higgs different, i.e. why is the Higgs soft mass much lower than typical superpartner masses? We do not pursue this avenue in this paper and instead allow that $\mathrm{TeV}$ size superpartner masses are generated at high scales.

The other possibility is to treat the Higgs differently from the superpartners by making it a pseudo-NambuGoldstone boson as in little Higgs theories [3] but now in the context of supersymmetry [4-8]. In this approach, radiative corrections to the Higgs soft mass are finite because they are "doubly protected" by softly broken supersymmetry and by the little Higgs mechanism. At tree-level the soft mass of a doubly protected Higgs vanishes, while the dominant radiative correction has the form

$$
\begin{aligned}
\delta m_{H}^{2} \approx & -\frac{3 y_{t}^{2}}{8 \pi^{2}}\left[\left(m_{\tilde{t}}^{2}+m_{T}^{2}\right) \log \left(m_{\tilde{t}}^{2}+m_{T}^{2}\right)-m_{\tilde{t}}^{2} \log \left(m_{\tilde{t}}^{2}\right)\right. \\
& \left.-m_{T}^{2} \log \left(m_{T}^{2}\right)\right],
\end{aligned}
$$

where $m_{T}$ is the mass of the little Higgs partner of the top. In the limit in which the little Higgs partners are much heavier than the superpartners, this formula reduces to Eq. (1) with $\Lambda_{\mathrm{UV}}$ replaced by $m_{T}$.

\footnotetext{
${ }^{1}$ Increasing the Higgs quartic coupling with new contributions from an extended Higgs sector without reducing the soft mass leads to new fine-tuning problems as was recently emphasized in [1].
} 
While the idea of double protection is very simple, explicit implementation encounters several problems. The original model of Birkedal et al. [4] relied on enlarging the standard model $S U(2)_{\text {weak }}$ into a global $S U(3)$ symmetry by introducing Higgs and top partners which complete $S U(3)$ triplets. The problem with this approach is that the standard mode gauge interactions do not respect the global $S U(3)$ symmetry. The renormalization group running from $\Lambda_{\mathrm{UV}}$ down to the weak scale badly breaks the $S U(3)$ symmetry so that it can no longer protect the soft Higgs mass. Note that this problem affects models which rely on a global symmetry which is explicitly broken by gauge interactions [e.g. a supersymmetry (SUSY) version of the littlest Higgs].

More recent attempts at implementing double protection therefore extend the global symmetry to the gauge interactions. In Refs. [6-8] the gauged $S U(2)_{\text {weak }}$ is enlarged to a gauged $S U(3)_{\text {weak }}$ as in the simplest little Higgs [9]. The pseudo-Nambu-Goldstone bosons (pNGBs) then arise because the $S U(3)_{\text {weak }}$ gauge symmetry is broken spontaneously to $S U(2)$ by two different sets of fields. If the coupling between these two sets of fields is sufficiently weak, then the theory has an approximate $S U(3)^{2}$ symmetry which is spontaneously broken to $S U(2)^{2}$, yielding two sets of pNGBs, one linear combination is eaten by the heavy $S U(3)_{\text {weak }}$ gauge bosons, the other remains light. A general problem with this approach is that the $S U(3)_{\text {weak }}$ Dterms strongly couple the two sectors and explicitly break the two $S U(3)$ 's of the Higgs sector to a single $S U(3)$. The would-be pNGBs get a mass from this D-term, and more model building is required to prevent it. Therefore realistic models end up being rather complicated.

In this paper we try a new approach based on the twin Higgs idea $[10,11]$ in which the global symmetry protecting the Higgs potential arises as an accidental symmetry after imposing a much more modest $Z_{2}$ twin parity. This spontaneously broken accidental symmetry makes the Higgs a pNGB and ensures double protection. An advantage of this idea is that it is relatively easy to implement the $Z_{2}$ symmetry by enlarging the field content of the MSSM.

The model we study first is left-right symmetric with the gauge group $S U(3)_{C} \times S U(2)_{L} \times S U(2)_{R} \times U(1)_{X}$ as in [11]. The $Z_{2}$ symmetry interchanges the left and right $S U(2)$ gauge bosons. Furthermore, every MSSM field has its $Z_{2}$ partner. In particular, the Higgs sector consists of four multiplets: two "left" doublets $H_{u}$ and $H_{d}$ and two "right" doublets $\tilde{H}_{u}$ and $\tilde{H}_{d}$. The $Z_{2}$ symmetry imposed on the Higgs sector is sufficient to guarantee an accidental $S U$ (4) symmetry of the dimension 2 terms in the Higgs potential. Thus, even though the Yukawa interactions which renormalize the Higgs mass terms do not respect the full $S U(4)$ (even after imposing the $Z_{2}$ symmetry), the resulting corrections to the Higgs masses are automatically $S U(4)$ symmetric. This is how double protection is realized in this model. Divergent radiative corrections to soft masses do not lead to masses for the pNGB because they respect the full global symmetry.

Unfortunately, this minimal twin supersymmetric model shares a problem with the models based on the $S U(3)_{\text {weak }}$ group discussed above: some of the quartic couplings in the Higgs sector, in particular, the $S U(2)_{L} \times S U(2)_{R} \times$ $U(1)_{X}$ D-terms explicitly break the $S U(4)$ symmetry and lead to large tree-level masses for the would-be pNGBs. We demonstrate this problem in the minimal twin SUSY model in the next section. In Sec. III we show that a simple modification of the model can avoid the troublesome Dterm contributions and lead to a fully realistic model. Section IV contains our conclusions.

\section{A TOY MODEL FOR TWIN SUSY}

Our toy model, inspired by Ref. [11], is left-right symmetric with the gauge group $S U(3)_{C} \times S U(2)_{L} \times$ $S U(2)_{R} \times U(1)_{X}$. An additional discrete $Z_{2}$ symmetry interchanges the left and right $S U(2)$ gauge groups, thus enforcing $g_{L}=g_{R}$. The discrete symmetry requires that for every left doublet there exists a $Z_{2}$ partner (denoted by tilde) that transforms as a doublet under $S U(2)_{R}$. Therefore the minimal Higgs sector contains four multiplets: two left doublets $H_{u}$ and $H_{d}$ (required by anomaly cancellation) and two right doublets $\widetilde{H}_{u}$ and $\widetilde{H}_{d}$ (required by the $Z_{2}$ ). Their $U(1)_{X}$ charges are chosen as $+\frac{1}{2},-\frac{1}{2},-\frac{1}{2}$ and $+\frac{1}{2}$, respectively. With a help of a singlet superfield $N$ we can write a superpotential that yields interactions between the left and right Higgs doublets,

$$
W=\lambda N\left(H_{u} H_{d}+\tilde{H}_{u} \tilde{H}_{d}-F^{2}\right) .
$$

Note that the $Z_{2}$ symmetry ensures an accidental global $S U(4)$ symmetry $^{2}$ under which $\left(H_{u}, \tilde{H}_{u}\right)$ transform as $\overline{\mathbf{4}}$ and $\left(H_{d}, \tilde{H}_{d}\right)$ as 4 . Furthermore, it requires the soft mass terms of the Higgses to be $S U(4)$ symmetric

$$
\mathcal{L}_{\text {soft }}=-M_{u}^{2}\left(\left|H_{u}\right|^{2}+\left|\tilde{H}_{u}\right|^{2}\right)-M_{d}^{2}\left(\left|H_{d}\right|^{2}+\left|\tilde{H}_{d}\right|^{2}\right) .
$$

The linear term in Eq. (3) forces some of the Higgs fields to acquire vacuum expectation value (VEVs). In Sec. III we introduce explicit $S U(4)$ breaking interactions which are responsible for generating a quartic coupling for the physical Higgs boson and which determine the VEV to lie in the $\tilde{H}_{u, d}$ direction. We parametrize $\left\langle\tilde{H}_{u}\right\rangle=f \sin \beta,\left\langle\tilde{H}_{d}\right\rangle=$ $f \cos \beta$, where $f$ depends on $F$ and the soft masses and $\tan ^{2} \beta=\left(M_{d}^{2}+\lambda^{2} N^{2}\right) /\left(M_{u}^{2}+\lambda^{2} N^{2}\right)$. The $S U(4)$ symmetry is spontaneously broken down to $S U(3)$ yielding 7 Nambu-Goldstone bosons. Three are eaten as a result of gauge symmetry breaking $S U(2)_{R} \times U(1)_{X} \rightarrow U(1)_{Y}$, leaving four physical NGBs. These form an $S U(2)_{L}$ doublet $H$ that is identified with the SM Higgs field. In the

\footnotetext{
${ }^{2}$ Emergence of $S U(4)$ symmetry in such a setup was also noted in Ref. [12].
} 
nonlinear sigma model parametrization,

$$
\begin{aligned}
& H_{u} \rightarrow f \sin \beta \sin (|H| / f) \frac{H}{|H|} \\
& H_{d} \rightarrow f \cos \beta \sin (|H| / f) \frac{\epsilon H^{*}}{|H|} \\
& \tilde{H}_{u} \rightarrow f \sin \beta \cos (|H| / f)\left(\begin{array}{c}
0 \\
1
\end{array}\right) \\
& \tilde{H}_{d} \rightarrow f \cos \beta \cos (|H| / f)\left(\begin{array}{l}
1 \\
0
\end{array}\right) .
\end{aligned}
$$

Note that $\tan \beta$ for the MSSM Higgs fields $H_{u}$ and $H_{d}$ is equal to the ratio of the VEVs of the heavy Higgses $\tilde{H}_{d}$ and $\tilde{H}_{u}$. With this parametrization it is easy to verify explicitly that neither the F-term potential nor the soft terms depend on the SM Higgs field $H$.

On the other hand, gauge and Yukawa interactions break $S U$ (4) explicitly, even after imposing the $Z_{2}$ symmetry. Therefore these interactions generate a potential for the SM Higgs at loop level. However, the $Z_{2}$ is sufficient to ensure double protection. The point is that $Z_{2}$ implies $S U(4)$ symmetry of all quadratic terms. Since the coefficient of the UV logarithm in the loop induced Higgs potential is quadratic in the Higgs fields (and quadratic in the supersymmetry breaking mass parameters), we are guaranteed it does not depend on the SM Higgs field $H$.

Renormalizable and $Z_{2}$ symmetric Yukawa interactions can be realized in our setup by introducing $Z_{2}$ partners for each MSSM matter multiplet (including "right-handed" neutrinos). The $S U(3)_{C} \times S U(2)_{L} \times S U(2)_{R} \times U(1)_{X}$ representation of the MSSM fields and their partners are given by (see also [13])

$$
\begin{array}{lc}
Q \rightarrow(3,2,1)_{1 / 6} & \tilde{Q}^{c} \rightarrow(\overline{3}, 1,2)_{-1 / 6} \\
L \rightarrow(1,2,1)_{-1 / 2} & \tilde{L}^{c} \rightarrow(1,1,2)_{1 / 2} \\
T^{c} \rightarrow(\overline{3}, 1,1)_{-2 / 3} & \tilde{T} \rightarrow(3,1,1)_{2 / 3} \\
B^{c} \rightarrow(\overline{3}, 1,1)_{1 / 3} & \tilde{B} \rightarrow(3,1,1)_{-1 / 3} \\
\tau^{c} \rightarrow(1,1,1)_{1} & \tilde{\tau} \rightarrow(1,1,1)_{-1} \\
N_{\tau}^{c} \rightarrow(1,1,1)_{0} & \tilde{N}_{\tau} \rightarrow(1,1,1)_{0},
\end{array}
$$

where the $U(1)_{X}$ charges are determined from the corresponding hypercharges using the identification $Y=X-$ $T_{R}^{3}$. The Yukawa interactions can be written as

$$
\begin{aligned}
W= & Y^{t} H_{u} Q T^{c}+Y^{t} \tilde{H}_{u} \tilde{Q}^{c} \tilde{T}+M^{t} \tilde{T} T^{c}+Y^{b} H_{d} Q B^{c} \\
& +Y^{b} \tilde{H}_{d} \tilde{Q}^{c} \tilde{B}+M^{b} \tilde{B} B^{c}+Y^{\tau} H_{d} L \tau^{c}+Y^{\tau} \tilde{H}_{d} \tilde{L}^{c} \tilde{\tau} \\
& +M^{\tau} \tilde{\tau} \tau^{c}+Y^{n} H_{u} L N_{\tau}^{c}+Y^{n} \tilde{H}_{u} \tilde{L}^{c} \tilde{N}_{\tau}+M^{n} \tilde{N}_{\tau} N_{\tau}^{c}
\end{aligned}
$$

We now compute the one-loop contribution to the SM Higgs potential due to the top sector. For simplicity, we assume degenerate soft masses for stops $\left(m_{\tilde{t}}^{2}\right)$ and vanishing A-terms

$$
\begin{aligned}
\delta V= & \delta m_{H}^{2}|H|^{2}+\delta \lambda|H|^{4}+\cdots \\
\delta m_{H}^{2} \approx & -\frac{3}{8 \pi^{2}} y_{t}^{2}\left[\left(m_{\tilde{t}}^{2}+m_{T}^{2}\right) \log \left(m_{\tilde{t}}^{2}+m_{T}^{2}\right)\right. \\
& \left.-m_{\tilde{t}}^{2} \log \left(m_{\tilde{t}}^{2}\right)-m_{T}^{2} \log \left(m_{T}^{2}\right)\right] \\
\delta \lambda \approx & \frac{3}{16 \pi^{2}} y_{t}^{4}\left[\log \left(\frac{m_{T}^{2} m_{\tilde{t}}^{2}}{\left(m_{\tilde{t}}^{2}+m_{T}^{2}\right) m_{t}^{2}}\right)+\frac{3}{2}\right. \\
& \left.-2 \frac{m_{\tilde{t}}^{2}}{m_{T}^{2}} \log \left(\frac{m_{\tilde{t}}^{2}+m_{T}^{2}}{m_{\tilde{t}}^{2}}\right)\right] .
\end{aligned}
$$

Here $m_{T}^{2}=\left(Y^{t} f \sin \beta\right)^{2}+\left(M^{t}\right)^{2}$ is the mass squared of the heavy top partner and $y_{t}=\left(Y^{t} \sin \beta\right)^{2} f / m_{T}$ is the SM top Yukawa coupling. These formulas are valid up to $\mathcal{O}(v / f)^{2}$ corrections. Note that the contribution to the SM Higgs mass is negative and can trigger electroweak symmetry breaking. The correction to the quartic terms is similar as in the MSSM, for large $m_{T}^{2}$ or large $m_{\tilde{t}}^{2}$ it becomes $\delta \lambda \rightarrow$ $\left(3 y_{t}^{4} / 16 \pi^{2}\right) \log \left[\min \left(m_{\tilde{t}}^{2}, m_{T}^{2}\right) / m_{t}^{2}\right]$.

Unfortunately, this nice and economical model is not viable. The problem is very similar to that which arises in gauged $S U(3)_{\text {weak }}$ models [6-8]. The $S U(2)_{L} \times S U(2)_{R} \times$ $U(1)_{X}$ D-term potentials do not respect the $S U(4)$ global symmetry and, in general, yield a tree-level mass for the SM Higgs. The effects of $S U(4)$ breaking are easy to understand in the limit $F>M_{\text {soft }}$ which is required by lower bounds on the masses of the $S U(2)_{R} \times U(1)_{X}$ gauge bosons from LEP experiments. After integrating out the fields with masses of order $F$ and to lowest order in $M_{\text {soft }}^{2} / F^{2}$, the potential for the SM Higgs has the form $V=$ $V_{\text {soft }}+V_{\text {quartic }}$, where

$$
\begin{aligned}
V_{\text {soft }}= & |H|^{2}\left[\frac{M_{u}^{2}+M_{d}^{2}}{2}(\cos \beta-\sin \beta)^{2}\right. \\
& \left.+\frac{M_{u}^{2}-M_{d}^{2}}{2}\left(1+\frac{g_{Y}^{2}}{g_{L}^{2}}\right) \cos ^{2} 2 \beta\right] \\
V_{\text {quartic }}= & |H|^{4}\left[\frac{g_{L}^{2}+g_{Y}^{2}}{8} \cos ^{2} 2 \beta\right] .
\end{aligned}
$$

We see that avoiding a large tree-level soft mass for the Higgs requires $M_{u}^{2}=M_{d}^{2}$ and, in consequence, $\sin \beta=$ $\cos \beta$, i.e. $\tan \beta=1$. But then the tree-level quartic vanishes as well which is in conflict with the lower bound on the Higgs mass from LEP2. The vanishing of the Higgs soft mass at $\tan \beta=1$ can be understood by noting that for $\tan \beta=1$ the D-terms have vanishing expectation values. Therefore the D-term potentials are supersymmetric and can only give a mass to the superpartners of the eaten NGBs, but not to the light Higgs doublet.

\section{A REALISTIC MODEL}

The problem with the toy model of the previous section is that the $S U(2)_{R} \times U(1)_{X}$ D-terms do not respect the global $S U(4)$ which protects the Higgs from obtaining a mass. This implies that we either have a large soft mass for 
the Higgs $(\tan \beta \neq 1$ ) or a vanishing tree-level quartic for the Higgs ( $\tan \beta=1)$. Either choice is problematic.

To solve this problem, two basic strategies are possible. One is to choose $\tan \beta \neq 1$ and remove the offending Dterms by breaking the $S U(2)_{R} \times U(1)_{X}$ gauge symmetry at a higher scale with additional charged fields. ${ }^{3}$ Alternatively, we may enforce $\tan \beta=1$ with an approximate symmetry, and obtain a new tree-level contribution to the Higgs quartic from appropriate superpotential couplings. This is the route which we take in the following.

We wish to stabilize the VEVs of $\tilde{H}_{u, d}$ at $\tan \beta=1$ which requires $M_{u}^{2} \approx M_{d}^{2}$ because $\tan ^{2} \beta=\left(M_{d}^{2}+\right.$ $\left.\lambda^{2} N^{2}\right) /\left(M_{u}^{2}+\lambda^{2} N^{2}\right)$. We therefore impose an approximate $Z_{2}$ symmetry acting as $u \leftrightarrow d$. Contributions to the Higgs mass from Eq. (9) only appear at second order in the difference $M_{u}^{2}-M_{d}^{2}$ so that small radiative corrections to $M_{u}^{2}=M_{d}^{2}$ can be tolerated. In general, these corrections can be large due to one-loop diagrams involving the Yukawa couplings in the top and bottom sector. However, the $u \leftrightarrow d$ symmetry also implies $Y^{t} \approx Y^{b}$ in which case the radiative corrections respect $M_{u}^{2}=M_{d}^{2}$. Note that the top and bottom quark masses can be split by choosing $M^{b} \gg M^{t}$ in Eq. (7), this breaks $u \leftrightarrow d$ softly, and a small Higgs mass is generated.

Since $\tan \beta=1$ we need an additional contribution to the SM Higgs quartic term. Consider the model of the previous section with four additional singlets $S, \tilde{S}, S^{c}, \tilde{S}^{c}$ and the superpotential

$$
W=\kappa S\left(H_{u} H_{d}-M_{s} S^{c}\right)+\kappa \tilde{S}\left(\tilde{H}_{u} \tilde{H}_{d}-M_{s} \tilde{S}^{c}\right) .
$$

The purpose of the first superpotential term is to add a contribution to the quartic coupling of the Higgs from the F-term of $S$. In the absence of a soft mass, the field $S^{c}$ would adjust its VEV such as to cancel the quartic. We therefore require a sizable soft mass for $S^{c}$. The second superpotential term is required by the $Z_{2}$ symmetry. When expanded in terms of the light Higgs field this term can contribute a large soft mass to the Higgs. We therefore require a small soft mass for $\tilde{S}^{c}$ so that its VEV can adjust to cancel the Higgs soft mass. Thus, for our model to work we must explicitly break the $Z_{2}$ symmetry with the soft terms for $S^{c}$ and $\tilde{S}^{c}$ (alternatively, the breaking could be achieved by splitting the masses $M_{s}$ and $M_{\tilde{s}}$ ). This explicit breaking of the $Z_{2}$ in the soft masses is radiatively stable because the soft terms for $S^{c}$ and $\tilde{S}^{c}$ do not run at any loop order.

To determine the range of viable soft masses for $S^{c}$ and $\tilde{S}^{c}$, we write the contribution to the Higgs potential from Eq. (10) which involves $S^{c}$ and $\tilde{S}^{c}$ and their soft masses

\footnotetext{
${ }^{3}$ Alternatively, the D-terms can be removed with "supersoft" [14] Dirac mass terms for the $S U(2)_{R} \times U(1)_{X}$ gauginos [15].
}

$$
\begin{aligned}
V= & \kappa^{2}\left|M_{s} S^{c}-\frac{f^{2}}{2} \sin \left(\frac{|H|}{f}\right)\right|^{2}+m^{2}\left|S^{c}\right|^{2} \\
& +\kappa^{2}\left|M_{s} \tilde{S}^{c}-\frac{f^{2}}{2} \cos \left(\frac{|H|}{f}\right)\right|^{2}+\tilde{m}^{2}\left|\tilde{S}^{c}\right|^{2}
\end{aligned}
$$

and minimize the potential for the singlets. We find that a sufficiently large quartic requires $\kappa=\mathcal{O}(1), m \geqslant M_{s}$ and $\tilde{m} \ll M_{s}$. In this limit, the expressions for the Higgs soft mass and quartic are

$$
m_{H}^{2}=-\tilde{m}^{2} \frac{f^{2}}{2 M_{s}^{2}} \quad \lambda=\frac{\kappa^{2}}{4} \frac{m^{2}}{\kappa^{2} M_{s}^{2}+m^{2}} .
$$

Taking for example $\kappa^{2}=1 / 2, M_{s}=m=1 \mathrm{TeV}, f=$ $3 \mathrm{TeV}$ and $\tilde{m}=50 \mathrm{GeV}$ gives a sufficiently large treelevel quartic $(\lambda=1 / 12)$ and a contribution to the soft mass of order the Z-mass $\left(m_{H}=106 \mathrm{GeV}\right)$. Note that at the minimum of the potential $S$ and $N$ do not have scalar expectation values but that the $F$ component of $S$ is nonzero. Therefore Eq. (10) contains an effective $B \mu$ term for the Higgs but no $\mu$ term which is necessary in order to make the Higgsinos in $H_{u}$ and $H_{d}$ sufficiently heavy. To generate a Higgsino mass, we add the $Z_{2}$ [and $S U(4)$ ] symmetric term $\mu\left(H_{u} H_{d}+\tilde{H}_{u} \tilde{H}_{d}\right)$. Since the singlet $N$ has a soft mass term its VEV cannot adjust to cancel the $\mu$-term completely and Higgsino masses are generated.

\section{UNIFICATION}

The particle spectrum in Eq. (6) is suggestive of various steps of unification. First quarks and leptons may be unified into an $S U(4)$ Pati-Salam "color" group where the $U(1)_{X}$ charges arise as a combination of the $\operatorname{diag}(1,1,1,-3)$ generator of $S U(4)$ and "middle" $U(1)_{M}$.

The unified fields transform under $S U(4) \times S U(2)_{L} \times$ $S U(2)_{R} \times U(1)_{M}$ as

$$
\begin{aligned}
(Q, L) & \rightarrow(4,2,1)_{0} & \left(\tilde{Q}^{c}, \tilde{L}^{c}\right) \rightarrow(\overline{4}, 1,2)_{0} \\
\left(T^{c}, N_{\tau}^{c}\right) & \rightarrow(\overline{4}, 1,1)_{-1 / 2} & \left(\tilde{T}, \tilde{N}_{\tau}\right) \rightarrow(4,1,1)_{1 / 2} \\
\left(B^{c}, \tau^{c}\right) & \rightarrow(\overline{4}, 1,1)_{1 / 2} & (\tilde{B}, \tilde{\tau}) \rightarrow(4,1,1)_{-1 / 2} \\
H_{u} & \rightarrow(1,2,1)_{1 / 2} & \tilde{H}_{u} \rightarrow(1,1,2)_{-1 / 2} \\
H_{d} & \rightarrow(1,2,1)_{-1 / 2} & \tilde{H}_{d} \rightarrow(1,1,2)_{1 / 2} .
\end{aligned}
$$

This particle content suggests a further unification where $U(1)_{M}$ is the $T^{3}$ generator of a new $S U(2)_{M}$ with the field content $S U(4) \times S U(2)_{L} \times S U(2)_{M} \times S U(2)_{R}$

$$
\begin{aligned}
\Psi_{L} & =(Q, L) \rightarrow(4,2,1,1) \\
\Psi_{R}^{c} & =\left(\tilde{Q}^{c}, \tilde{L}^{c}\right) \rightarrow(\overline{4}, 1,1,2) \\
\Psi_{M}^{c} & =\left(T^{c}, N_{\tau}^{c}, B^{c}, \tau^{c}\right) \rightarrow(\overline{4}, 1,2,1) \\
\Psi_{M} & =\left(\tilde{T}, \tilde{N}_{\tau}, \tilde{B}, \tilde{\tau}\right) \rightarrow(4,1,2,1) \\
H & =\left(H_{u}, H_{d}\right) \rightarrow(1,2,2,1) \\
\tilde{H} & =\left(\tilde{H}_{u}, \tilde{H}_{d}\right) \rightarrow(1,1,2,2) .
\end{aligned}
$$


The Yukawa couplings now take a particularly simple form

$$
Y^{L} \Psi_{L} H \Psi_{M}^{c}+M \Psi_{M} \Psi_{M}^{c}+Y^{R} \Psi_{M} \tilde{H} \Psi_{R}^{c} .
$$

The "unification" presented here may also help in solving a problem with Landau poles for the $S U(3)_{c} \times U(1)_{X}$ gauge couplings. With all "twin" partners added, neither $S U(3)_{c}$ nor $U(1)_{X}$ are asymptotically free. Their respective Landau poles occur at $10^{13}$ and $10^{11} \mathrm{GeV}$ (if all extra fermion masses are at the $\mathrm{TeV}$ scale). Above $S U(4) \times$ $S U(2)_{M}$ breaking scale the running can be much slower due to the contribution of extra gauge bosons, provided the number of extra matter multiplets is small enough. Thus the Landau pole can be avoided assuming an $S U(4) \times$ $S U(2)_{M}$ breaking scale well below $10^{11} \mathrm{GeV}$. Note that this does not imply problems with proton decay as unification into $S U(4)$ does not violate baryon number. The situation here is somewhat better than in the $S U(3)_{\text {weak }}$ models, where perturbative unification is difficult to achieve [8]. However, the gauge couplings do not unify with the particle spectrum in Eq. (14), it would require additional matter multiplets. Furthermore, splitting of quark and lepton masses within generations is nontrivial, especially for $\tan \beta \approx 1$.

\section{CONCLUSIONS}

In this paper we constructed a supersymmetric model in which the Higgs is a pseudo-Nambu-Goldstone boson. Our model is left-right symmetric with the electroweak gauge group extended to $S U(2)_{L} \times S U(2)_{R} \times U(1)_{X}$. Imposing a $Z_{2}$ twin parity which interchanges the left and right $S U(2)$ 's implies an accidental $S U(4)$ symmetry of all mass terms in the extended Higgs sector. The Higgs
pNGB arises after spontaneous breaking of this $S U(4)$. The interplay of the global symmetry and supersymmetry leads to double protection: the Higgs mass parameter does not receive logarithmically divergent corrections at one loop, even in the presence of soft supersymmetry breaking. The reason is that at one loop only dimension two terms can be logarithmically renormalized, but these are automatically $S U(4)$ symmetric by virtue of the $Z_{2}$.

The purpose of this construction is a solution to the supersymmetric little hierarchy problem. Removing the one-loop logarithmic divergence allows for $M_{\text {SUSY }} \sim$ $4 \pi M_{W}$ without fine-tuning. Our model provides an explicit realization of double protection which is stable under radiative corrections. Technical complications related to the $S U(2)_{R} \times U(1)_{X}$ D-terms which do not respect the global symmetry and contribute to the pNGB mass at tree-level force us to introduce an extended Higgs sector. Thus, our solution to the little hierarchy problem in the MSSM comes at the price of simplicity-several new gauge singlets with carefully designed interactions are required at the $\mathrm{TeV}$ scale. The idea of stabilizing little hierarchy by global symmetries deserves further study, both at the theoretical and at the phenomenological level.

\section{ACKNOWLEDGMENTS}

M. S. thanks CERN Theory for hospitality and T. Roy for comments on the manuscript. A.F. and S.P. were partially supported by the European Community Contract No. MRTN-CT-2004-503369 for years 2004-2008 and by the MEiN Grant No. 1 P03B 09929 for years 2005-2007. M. S. acknowledges support from DOE Grant No. DEFG02-91ER40676.
[1] P. C. Schuster and N. Toro, hep-ph/0512189.

[2] K. Choi, K. S. Jeong, T. Kobayashi, and K.i. Okumura, Phys. Lett. B 633, 355 (2006); R. Kitano and Y. Nomura, Phys. Lett. B 631, 58 (2005).

[3] N. Arkani-Hamed, A. G. Cohen, and H. Georgi, Phys. Lett. B 513, 232 (2001); N. Arkani-Hamed, A. G. Cohen, E. Katz, A.E. Nelson, T. Gregoire, and J.G. Wacker, J. High Energy Phys. 08 (2002) 021; N. Arkani-Hamed, A. G. Cohen, E. Katz, and A. E. Nelson, J. High Energy Phys. 07 (2002) 034. For a review, see M. Schmaltz and D. Tucker-Smith, hep-ph/0502182; M. Perelstein, hep-ph/0512128.

[4] A. Birkedal, Z. Chacko, and M. K. Gaillard, J. High Energy Phys. 10 (2004) 036.

[5] P. H. Chankowski, A. Falkowski, S. Pokorski, and J. Wagner, Phys. Lett. B 598, 252 (2004).

[6] Z. Berezhiani, P.H. Chankowski, A. Falkowski, and S. Pokorski, Phys. Rev. Lett. 96, 031801 (2006).
[7] T. Roy and M. Schmaltz, J. High Energy Phys. 01 (2006) 149.

[8] C. Csaki, G. Marandella, Y. Shirman, and A. Strumia, Phys. Rev. D 73, 035006 (2006).

[9] D. E. Kaplan and M. Schmaltz, J. High Energy Phys. 10 (2003) 039.

[10] Z. Chacko, H. S. Goh, and R. Harnik, Phys. Rev. Lett. 96, 231802 (2006).

[11] Z. Chacko, H. S. Goh, and R. Harnik, J. High Energy Phys. 01 (2006) 108.

[12] Z. Berezhiani, hep-ph/0508233.

[13] Z. G. Berezhiani, Phys. Lett. 129B, 99 (1983); Yad. Fiz. 42, 1309 (1985) [Sov. J. Nucl. Phys. 42, 825 (1985)].

[14] P. J. Fox, A. E. Nelson, and N. Weiner, J. High Energy Phys. 08 (2002) 035.

[15] S. Chang, L. J. Hall, and N. Weiner, hep-ph/0604076; Seminar at Boston University, 2005. 